This item was submitted to Loughborough's Research Repository by the author.

Items in Figshare are protected by copyright, with all rights reserved, unless otherwise indicated.

\title{
Modification of the Internal Flows of Thermal Propulsion Systems Using Local Aerodynamic Inserts
}

PLEASE CITE THE PUBLISHED VERSION

https://doi.org/10.4271/2020-01-2039

PUBLISHER

SAE International

VERSION

AM (Accepted Manuscript)

PUBLISHER STATEMENT

This paper was accepted for publication in the journal SAE Technical Papers and the definitive published version is available at https://doi.org/10.4271/2020-01-2039.

LICENCE

CC BY-NC-ND 4.0

\section{REPOSITORY RECORD}

Butcher, Daniel, John Gillespie, Duncan Walker, K Todd Lowe, and Adrian Spencer. 2020. "Modification of the Internal Flows of Thermal Propulsion Systems Using Local Aerodynamic Inserts". Loughborough University. https://hdl.handle.net/2134/12993500.v1. 


\title{
Modification of the Internal Flows of Thermal Propulsion Systems using Local Aerodynamic Inserts
}

\author{
Author, co-author (Do NOT enter this information. It will be pulled from participant tab in \\ MyTechZone) \\ Affiliation (Do NOT enter this information. It will be pulled from participant tab in MyTechZone)
}

\begin{abstract}
Modern thermal propulsion systems (TPS) as part of hybrid powertrains are becoming increasingly complex. They have an increased number of components in comparison to traditionally powered vehicles leading to increased demand in packaging requirements. Many of the components in these systems relate to achieving efficiency gains, weight saving and pollutant reduction. This includes turbochargers and diesel or gasoline particulate filters for example and these are known to be very sensitive to inlet boundary conditions. When overcoming packaging requirements, sub-optimal flow distributions throughout the TPS can easily occur. Moreover, the individual components are often designed in isolation assuming relatively flat and artificially quiescent inlet flow conditions in comparison to those they are actually presented with. Thus, some of the efficiency benefits are lost through reduced component aerodynamic efficiency. Using local aerodynamic modification devices, StreamVane ${ }^{\mathrm{TM}}$, to eliminate excessive secondary velocity components prior to TPS component inlet boundaries is considered one solution to this. This type of device has been previously demonstrated to be effective at generating a wide range of secondary velocity profiles. With minor modifications, it should be possible to use these devices to instead remove secondary velocities. As a demonstration, a vane-pack designed to generate a counter-rotating vortex pair in a duct from a uniform flow is considered. An investigation is then carried out using ReynoldsAveraged Navier-Stokes (RANS) numerical simulation to assess the effectiveness of the same device in removing vorticity and secondary velocity components when presented with its design outflow in reverse, i.e. producing uniform flow when presented with a complex inlet flow. Results presented demonstrate clear potential for a vane pack to be used to produce a uniform flow thereby addressing the TPS packaging problem. Without any design modification for reverse flow, the insert reduced the mean secondary velocity magnitude by around $75 \%$ without a notable increase in system pressure loss. Consequently, proof-of-concept has been achieved and the use of a generic vane pack design process provides a realistic solution worthy of further development
\end{abstract}

\section{Introduction}

Thermal propulsion systems (TPS) / internal combustion engines (ICEs) remain the dominant powerplant in the automotive industry. According to Senecal and Leach [1] they power over $99 \%$ of the one billion passenger vehicles in use globally. Whilst the automotive industry has continually innovated and developed over several decades, leading to orders of magnitude reduction in some tail-pipe specific emissions, there is rightly the need to continue seeking efficiency improvements in our transport sector.

Various government mandated phase-outs of fossil fuels around the world has to date been agreed by 13 countries and 20 cities, with a range of measures proposed, mostly focusing on battery electric vehicles or hybrid battery and TPS powertrains. For example, in the United Kingdom, the Government proposes to introduce a ban from 2035 on the sale of conventional powered vehicles in the passenger car market. Even those powertrains that remain with a TPS component must improve efficiency (brake thermal efficiency). Targets set by the Automotive Council UK [2] state improvement in the light- and heavy-duty markets to $48 \%$ and $55 \%$ respectively by 2025 and $53 \%$ and $60 \%$ respectively by 2035 .

This presents a new landscape for the development of TPS powertrains, which will certainly include a battery electric element in passenger vehicles, increasing the complexity and packaging requirements. New ICEs must be developed in-parallel to be cleaner and more efficient, whilst being tolerant to potential sub-optimal routing of inlet and exhaust gases. Further, with increased electrification and complexity of the powertrain comes increasing cost. Therefore there is a great focus in ICE research to improve the efficiency without significant increases in purchase and operating costs [3].

In order to achieve these efficiency improvements and associated emissions reductions, it is recognized that several technological improvements to the TPS must be incorporated. These include electrified turbochargers which, according to Zhao et al. [4], have the potential to improve fuel economy by $10 \%$, as well as exhaust waste heat recovery systems which use thermoelectric generation (Yang et al. [5]). However, as these technologies rely on exhaust gas flows, their performance can be sensitive to boundary conditions. A further important technology identified as being sensitive to aerodynamic inlet conditions is reactive aftertreatment systems. Selective catalytic reduction (SCR) is vital in meeting EURO6 emissions in both lightand heavy-duty vehicle classes. Qi et al. [6] state that this is of particular importance with the increasing use of biodiesel. These devices, and similar such as diesel and gasoline particulate filters, are positioned in the exhaust stream and are known to be highly sensitive to inflow conditions. For example, Merati et al. [7] suggest that optimal conversion efficiency in SCRs is achieved when the exhaust gas approaches in a uniformly distributed condition. This condition

Page 1 of 9

$10 / 19 / 2016$ 
makes use of the entire inlet area and provides the minimum temperature gradients across the monolith. In significant cases of maldistribution, deposits may be increased and premature aging of the catalyst material may occur.

Diffusers or sudden expansions prior to the monolith are typically used to assist the uniform gas distribution, particularly where packaging requirements necessitate the use of bends close to the catalyst inlet. For example, in the work by work by Turner et. al. [8] stereoscopic Particle Image Velocimetry is used in an optically accessible catalyst 'inlet cone' to assess the profile of axial velocity uniformity with this approach. However, these approaches can often be difficult to realize in applications where packaging restrictions are particularly tight.

In addition to axial velocity uniformity, Persoons et. al. $[9,10]$ describes the effect of secondary flow velocities, in particular tangential velocity, on the inlet losses of a substrate. In conditions with a high tangential velocity component at inlet, flow separation at an oblique entrance causes local increased resistance. Benjamin et. al. $[11,12]$ show how the increased pressure drop due to tangential velocity, $K_{\alpha}$ in Equation 1 is proportional to the square of velocity magnitude.

$$
\Delta p=\left(\frac{4 f_{a p p} L}{d}+K_{c o n}+K_{e x p}+K_{\alpha}\right) \rho U_{C}^{2} / 2
$$

It is therefore a worthwhile objective to minimize the secondary flow velocities at the inlet face of any sensitive device in order to reduce net pressure cost over such a device.

\section{Scope of Investigation}

This paper will detail a preliminary investigation into the use of a novel, low-cost, local aerodynamic insert, StreamVane ${ }^{\mathrm{TM}}$ [13]. Used to modify flow velocity distribution and properties in a duct typical of that found prior to the aftertreatment components of an automotive TPS. The purpose of modifications is to reduce secondary flow magnitudes, increase flow uniformity and hence improve the efficiency or durability of a theoretical downstream component and/or to reduce the net pressure cost (drop) associated with it. A numerical methodology is used to assess the effectiveness of using an insert to reduce vorticity (removing secondary flows) and improving uniformity. This is of interest for example in application prior to a catalytic converter or gasoline or diesel particulate filter, (GPF / DPF) either from the design and development stage, or as a retrofit upgrade to an existing fleet. As stated in the introduction, the filter performance would be enhanced, and the net pressure loss potentially reduced with the vane insert designed to remedy any flow disturbances generated by upstream components. Specific findings of the research presented may be utilized further in the development of aerodynamic modifying inserts, designed specifically for the purpose of producing either uniform flow distribution, or the removal of secondary velocities.

\section{StreamVane Method}

A process for designing an aerodynamic insert to produce a swirling flow has previously been demonstrated to be effective for generating various different swirl profiles [13]. Each device is designed uniquely to produce a particular swirling flow, depending on the application; for example, one device may generate a single vortex, another, two symmetric vortices, and another, a pattern applicable to a complex serpentine duct. By demonstrating that the device can also be used to remove secondary velocities (rather than generating them), it should be possible to extend the existing method to produce tailored devices for the removal of particular swirls. As with the standard device, each design would be created with the goal of removing a particular swirl profile. For example, the secondary velocities expected to be generated within an automotive TPS. The (standard) StreamVane design process proceeds in the following steps:

- Determine the target swirl profile, defined at a particular plane within the duct. This plane is known as the aerodynamic interface plane (AIP)

- Solve the inverse vorticity transport problem to create a design swirl profile for the StreamVane exit plane. This design profile is the profile that, when produced at the trailing edges of the vane pack, results in the correct target swirl profile at the AIP

- Design a 3D vane pack (StreamVane) to produce the required swirl angles at the trailing edge

- Using low order performance models, improve the design by making incremental changes

- Verify that the design is appropriate by running a full 3D CFD analysis

The original StreamVane design process is described by Hoopes and O'Brien [14].Various improvements have been developed since then, but it is at its core the same technique.

StreamVanes are most commonly manufactured by additive manufacturing, particularly at small scales. As TPS systems are often subject to significant temperatures, a metallic device is likely necessary. Production of a StreamVane at 2-inch $(50.8 \mathrm{~mm})$ scale is possible with a variety of additive techniques, including direct metal laser sintering (DMLS).

\section{Investigation Methodology}

The objective of the presented work is to develop understanding of how a local aerodynamic insert may be used to remove or reduce non-uniform secondary flow velocity content from a duct flow; providing preliminary information for the development of a dedicated StreamVane device for this purpose in automotive TPS flows. The high-level approach will be discussed in this section, before detailed methodologies are described as necessary.

For all the presented work, a single StreamVane device has been used; a detailed description is given in the following subsection. In summary, the device is designed to take uniform axial flow with no secondary velocity components - generating a symmetrical counterrotating vortex pair in the duct after the insert. A CFD simulation is carried out with a uniform axial velocity inlet, and the downstream flow development is studied.

At several XY-plane locations downstream of the insert (where $\mathrm{Z}$ is the axial flow direction and $X Y$ represents the secondary flow distribution), the 3-component velocity profile is extracted together with the turbulence intensity distribution. These are then reversed and taken as the inlet conditions of a further series of simulations, i.e. with the swirling flow approaching the vanepack. In each condition, the effectiveness of the insert at removing the secondary velocity and vorticity content may be assessed. 


\section{Stream Vane Insert Design}

The StreamVane was designed to produce symmetric twin vortex pair presented in Figure 1. It is worth noting that most swirl distortions undergo some, often significant, changes as they propagate downstream due to vortex dynamics. So that the effect of running the reversed device could be isolated from the natural variation of the swirl profile, the specific profile chosen was a Lamb-Chaplygin dipole $[15,16]$. This has the distinction that it undergoes negligible change in magnitude or shape due to inviscid vortex dynamics. Thus, the only effects that can cause changes in the flow profile (in absence of the reversed StreamVane) are viscosity and turbulent diffusion, both of which are generally much weaker than the vortex dynamics.

Choosing this profile therefore allows a more direct observation of the effects of the reversed StreamVane than a profile that would change significantly on its own. This profile also enables step 2 in the design process to be skipped. For a detailed discussion of this type of minimally-developing swirl distortion profile, see Gillespie et al. [17]. The specific swirl profile is given by the following Tangential and Radial Flow Angles, (TFA and RFA):

$$
\begin{aligned}
& T F A=\operatorname{atan}\left(\frac{c_{\theta}}{c_{z}}\right)=\operatorname{atan}\left(A \frac{\rho_{11}}{D}\left[J_{2}\left(\frac{2 \rho_{11} r}{D}\right)-J_{0}\left(\frac{2 \rho_{11} r}{D}\right)\right] \cos (\theta)\right)(2) \\
& R F A=\operatorname{atan}\left(\frac{c_{r}}{c_{z}}\right)=\operatorname{atan}\left(-A J_{1}\left(\frac{2 \rho_{11} r}{D}\right) \frac{\sin (\theta)}{r}\right)
\end{aligned}
$$

In these equations, $J_{0}, J_{1}$, and $J_{2}$ are the zeroth-order, first-order, and second-order Bessel functions of the first kind, and $\rho_{11}$ is the smallest positive root of $J_{1}$. A is an arbitrary scaling factor, and is chosen in this case to produce a maximum tangential flow angle (TFA) of $20^{\circ}$.

The airfoil profile chosen for this design (Figure 1) used the camber profile of a NACA 63-series airfoil [18]. For ease of manufacturing at small scale, no thickness profile was used. Instead, a simple elliptical leading edge was applied to an otherwise constant thickness.
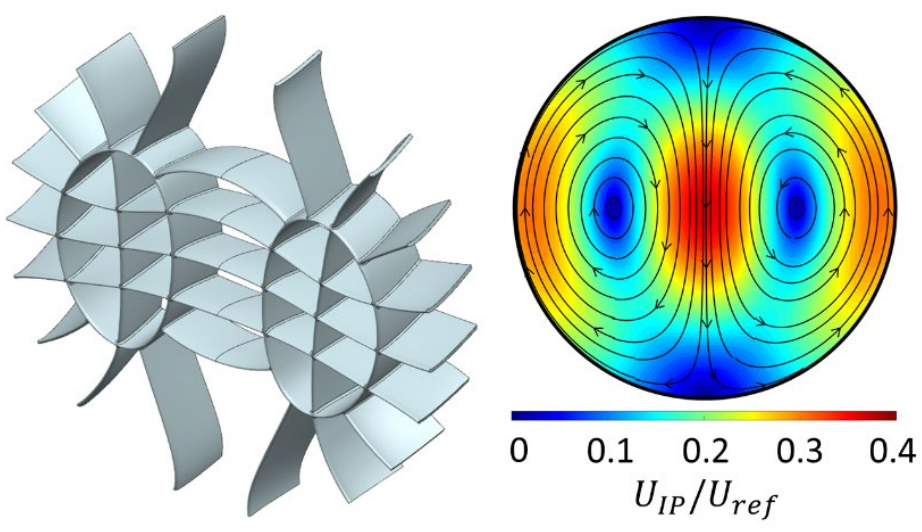

Figure 1. Twin-swirl geometry and design secondary velocity profile

\section{Computational Methodology}

The computation domain (Figure 2) consists of the vane pack positioned in a 2 -inch diameter $(50.8 \mathrm{~mm})$ pipe. For the forward flow case the inlet was positioned 2 diameters upstream of the vanes and the outlet 5 diameters downstream. For the reverse flow case the inlet condition was specified from velocity and turbulence data extracted from the forward flow case at several planes $(\mathrm{z} / \mathrm{D}=0.5,1.5,2.5,4.5)$.
Hence, for the reverse flow case the domain was modified to ensure the inlet plane was the appropriate distance upstream of the vane pack and all outlet planes maintained at a downstream distance of 5D.

All numerical predictions were performed using the commercial platform ANSYS Workbench 19.0 with grids generated using ANSYS Mesher and solution of the steady Reynolds Averaged Navier-Stokes (RANS) equations performed using ANSYS Fluent. An unstructured tetrahedral mesh with a nominal cell size of $1.2 \mathrm{~mm}$ was employed with the final mesh comprising of $28.8 \mathrm{~m}$ cells. Additionally, 20 inflation layers were employed from the vane surfaces to ensure a wall $\mathrm{y}+$ close to 1 such that the vane boundary layer was fully resolved. A first layer height of $0.005 \mathrm{~mm}$ and growth rate of 1.15 ensured at least 40 cells in each passage between blades. The flow is isothermal and low speed $(\mathrm{M}<0.1)$ and hence modelled as incompressible with pressure-velocity coupling achieved using the SIMPLE algorithm. Spatial discretization of the governing equations was performed using $2^{\text {nd }}$ order numerical schemes. Turbulence was modelled using k- $\omega$ SST and a low $\mathrm{y}+$ approach to resolve the boundary layer. For the forward flow case the inlet was set as a uniform velocity of $25 \mathrm{~ms}^{-1}$ which represents a Reynolds number, based on vane chord, of $1.44 \times 10^{4}$. The inlet turbulence was also set as uniform with a low turbulence intensity of $1 \%$ and a turbulent length scale of $2 \mathrm{~mm}$ for the forward flow case.

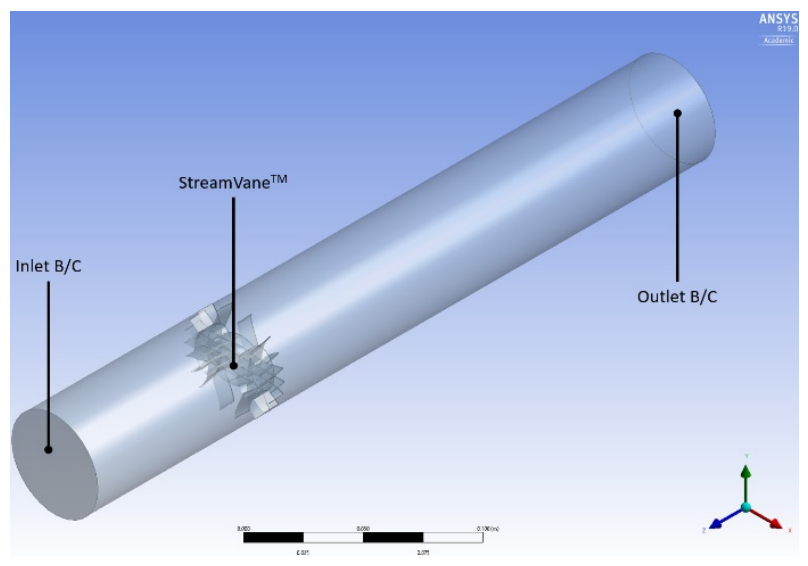

Figure 2. Computational domain used for forward flow simulation

A mesh sensitivity study was conducted by varying the global cell density with convergence to the final mesh assessed by examining the mass-weighted total pressure loss coefficient from inlet to exit. This is defined as:

$K=\frac{\tilde{P}_{i n}-\tilde{P}_{e x}}{\frac{1}{2} \rho U_{i n}^{2}}$

\section{Results: Forward Flow Direction}

Before numerical simulations of the reverse flow case are discussed, for context, the forward flow, or design case should be considered. As discussed in the previous section, the insert is designed to generate a counter-rotating, symmetrical vortex pair in the secondary plane (orthogonal to z-axis). In-plane velocity magnitude normalized

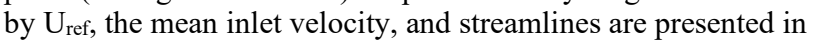
Figure 3 at three locations downstream of the insert; $0.5 \mathrm{D}, 1.5 \mathrm{D}$ and 4.5D from the trailing edge ( $\mathrm{a}, \mathrm{b}$ and c respectively). Note that it is this data that is used as the inlet condition for the reverse flowing cases.

Page 3 of 9 
From both Figure $3 \mathrm{~b}$ and $\mathrm{c}$, it can be observed that there is little to no development of the structures downstream. However, close to the trailing edge of the insert, within $0.5 \mathrm{D}$, there is visible evidence of wakes from each of the aerodynamic blades, signified by local increases in velocity magnitude in Figure 3a. These are however mixed out within $2 \mathrm{D}$ of the trailing edge.

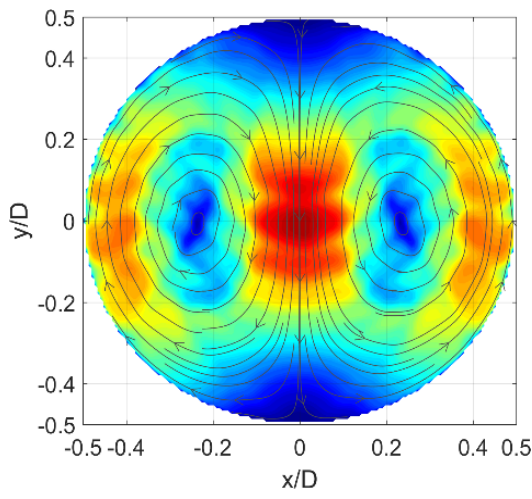

(a) $\quad \mathrm{z} / \mathrm{D}=-0.5$

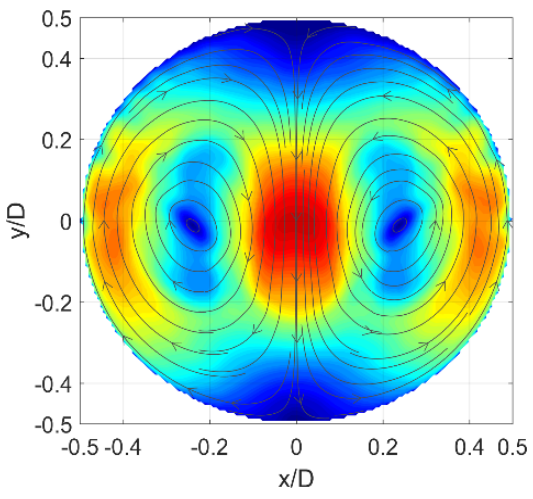

(b) $\mathrm{z} / \mathrm{D}=-1.5$

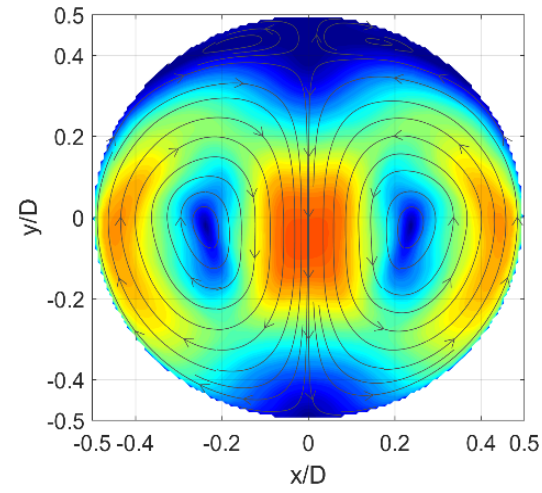

(c) $\mathrm{z} / \mathrm{D}=-4.5$

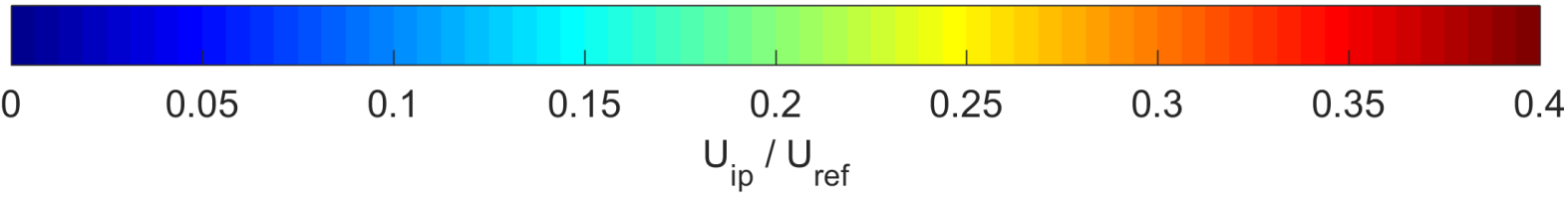

Figure 3. In-plane $(\mathrm{u}, \mathrm{v})$ normalized velocity magnitude and streamlines at $\mathrm{XY}$ planes at indicated $\mathrm{z}=$ constant positions downstream

At several points along the z-axis, the mass-weighted total and static pressure were evaluated, similar to that defined in Equation 4. Figure 4 shows how the pressure drop coefficient, $\mathrm{K}$ with reference to the inlet face condition develops. This grows along the length of the duct due to viscous losses in the near wall regions regardless of the vanepack. However, by considering the planes $0.5 \mathrm{D}$ up- and downstream, the total pressure drop coefficient over the vanepack is calculated as $\mathrm{K}=0.13$.

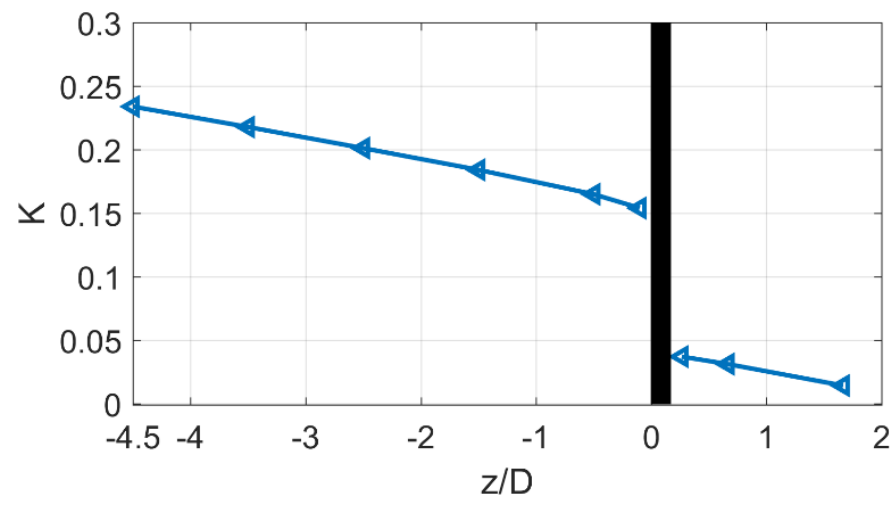

Figure 4. Total pressure drop coefficient, K (markers indicate flow direction)

The pressure drop has been considered at these locations (+/- 0.5D) rather than the leading and trailing edge planes to avoid calculations within a chord-length where turbulent, mixing losses are intense. Figure 5 shows the pressure drop distribution very close to the vanepack, determined from a pressure drop between axial planes at $+/-0.1 D$. Figure 5(a), the total pressure drop coefficient shows signatures of the vane wakes. In analysis of the static pressure drop coefficient, Figure 5(b), the large increase in vorticity across the vane Page 4 of 9 pack is evident by the local increase in coefficient which coincides with the location of the counter-rotating vortex pair.

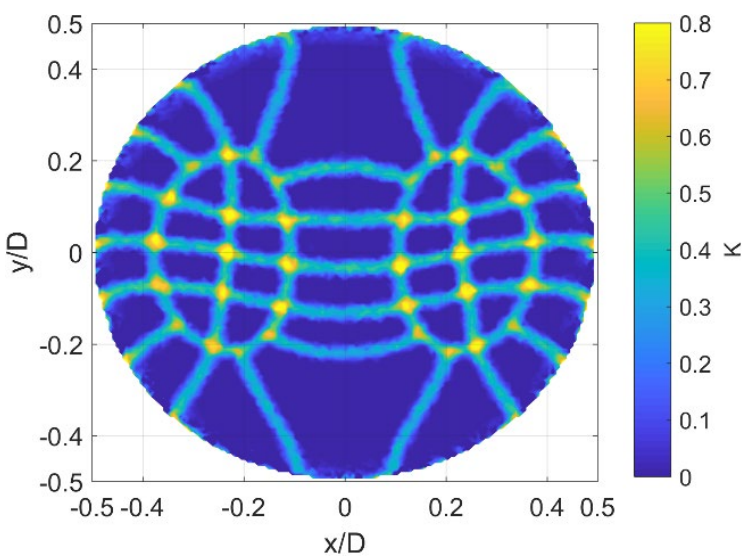

(a) Total pressure coefficient 


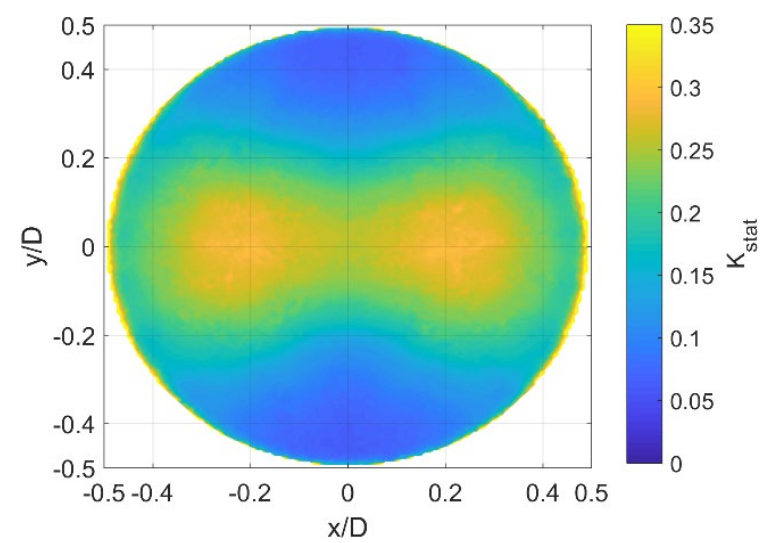

(b) Static pressure coefficient

Figure 5. Pressure drop coefficient distributions close to vane pack, +/- 0.1D

\section{Results: Reverse Flow Sweep}

Using the numerical simulation presented in the previous section, four planes are extracted downstream for the reverse flow study. The locations of these planes are described in Table 1.

Table 1. Definition of input planes for comparison of reverse flow sweep

\begin{tabular}{|l|l|}
\hline Inlet Plane & z/D \\
\hline Rev_A & -0.5 \\
\hline Rev_B & -1.5 \\
\hline Rev_C & -2.5 \\
\hline Rev_D & -4.5 \\
\hline
\end{tabular}

Similar methodology is followed in each of these cases, with only the mesh changed between each of the reverse flow cases. This was necessary to adjust the upstream geometry to be of the appropriate length to match the $\mathrm{z} / \mathrm{D}$ for each case. The remainder of this section discusses several summary results of the four cases.

Figure 6 presents the area-averaged secondary velocity magnitudes along the $z$-direction. To produce this figure, the magnitude of only the $\mathrm{u}-, \mathrm{v}$ - velocity components are averaged at $\mathrm{z}=$ constant planes along the length of the duct to show the development in each case.

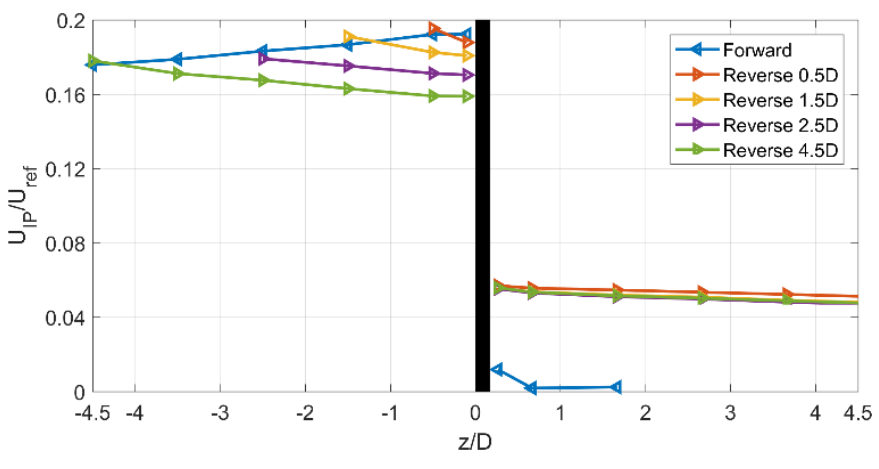

Figure 6. Development of area-averaged secondary velocity magnitude along duct (marker indicates flow direction)

Considering the forward flow case, which runs right to left in all appropriate figures (as indicated by the marker direction), there is initially very low secondary velocity upstream of the vane pack. This is because the inlet flow is defined as a uniform axial flow and there is only a small magnitude of secondary velocities in the near wall regions due to boundary layer development. Passing through the vanepack, the secondary velocity magnitude is increased significantly as would be expected due to the counter-rotating vortex pair (visible in Figure 3) which decays slightly along the length of the duct due to viscous losses.

Examining now each of the reverse flow cases, which flow left to right in all plots. These all start as expected from the forward flow line, where the planes were originally extracted, but immediately start to decay at similar rates before approaching the vanepack. Despite the decay rate being similar, as they have different lengths between inlet and the leading edge, the vanepack is presented with different velocity profiles and secondary velocity magnitudes in each case. Figure 7 presents examples for the velocity distribution at $0.5 \mathrm{D}$ upstream of the Rev_A, Rev_B and Rev_C cases (Figure 7(a) shows the reverse of Figure 3(a) as this is where the input is extracted for Rev_A). This illustrates the range of secondary velocities the vanepack is presented with.

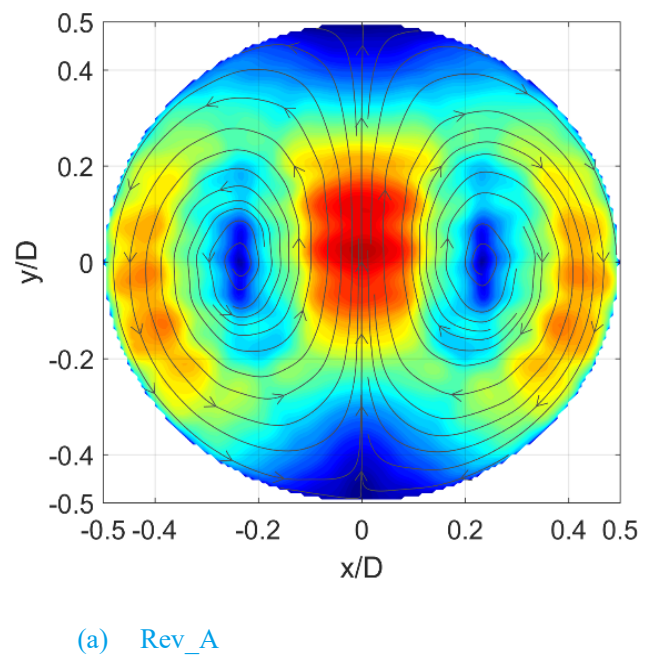




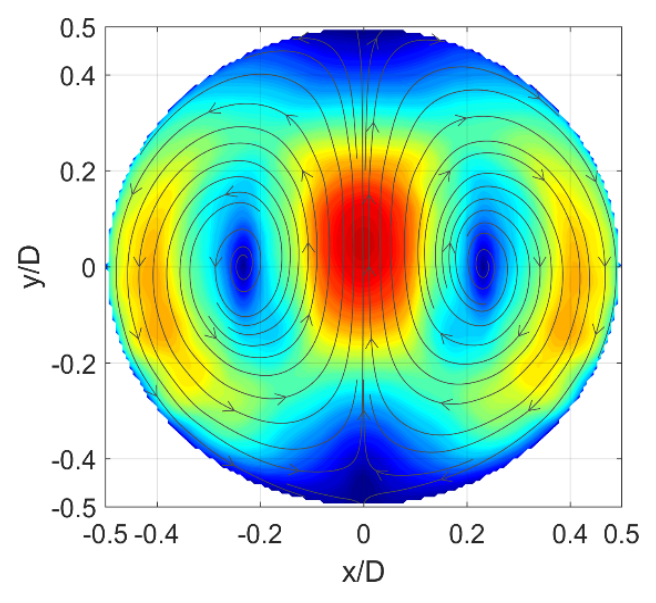

(b) Rev_B

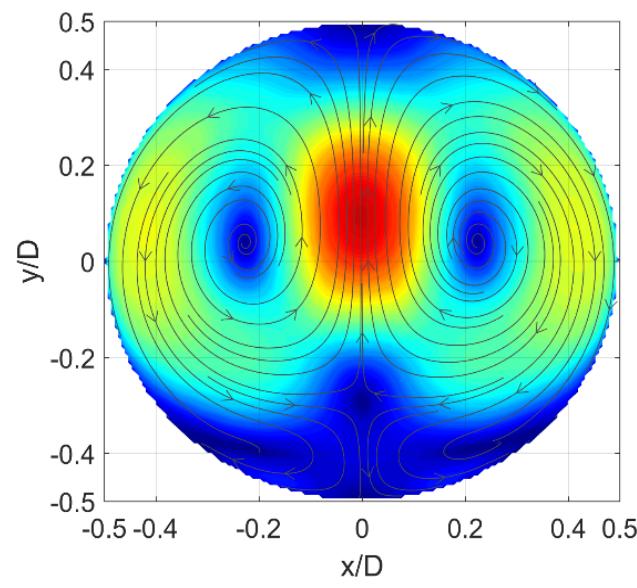

(c) Rev_D

Figure 7. In-plane velocity magnitude and streamlines at planes at $0.5 \mathrm{D}$ upstream from leading edge

It is noteworthy that despite being presented with different inlet conditions, Figure 6 shows how very similar resulting magnitude in the secondary velocity is seen downstream of the device. This suggests that the vanepack is robust to some level of variation about a nominal distribution in the inlet velocity. Whilst all the reverse cases tested maintain some secondary velocity components, the area averaged magnitude is reduced in all cases by approximately $75 \%$.

Thus far the work has been discussed in the context of applications to an automotive SCR with benefit being gained form the reduction of secondary velocity magnitudes. However, an improvement in flow uniformity would be also be beneficial. Hence, a uniformity index for velocity magnitude may be calculated according to Equation 5 to evaluate the effectiveness of producing a uniform distribution of velocity. (Note that a value of unity indicates complete uniformity).

$$
U I_{v}=1-\frac{\sum_{i=1}^{n} \frac{\sqrt{\left(v_{i}-\bar{v}\right)^{2}}}{\bar{v}} A_{i}}{2 A}
$$

The index is calculated on the same planes as previously and is presented in Figure 8. In all cases, there is evidence of an immediate decrease in uniformity within $2 \mathrm{D}$ of the vanepack, which recovers to a level which is similar to the upstream conditions.

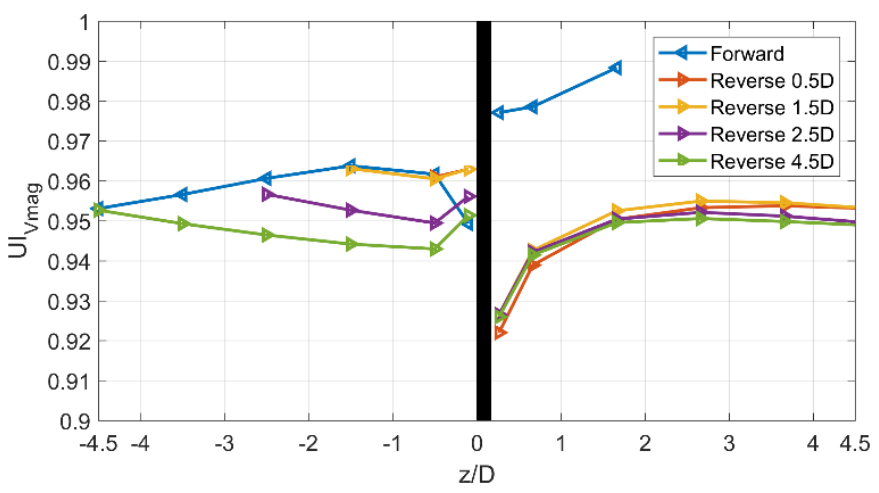

Figure 8. Development of uniformity index of velocity magnitude along duct (marker indicates flow direction)

The cause of both the initial decrease in uniformity and further characteristics can be explained by examining the immediate downstream velocity profile as presented in Figure 9 for the Rev_C case. At a plane very close to the trailing edge, $0.03 \mathrm{D}$, the large vortices have been removed, but smaller structures can be seen formed within the passages between individual vanes.

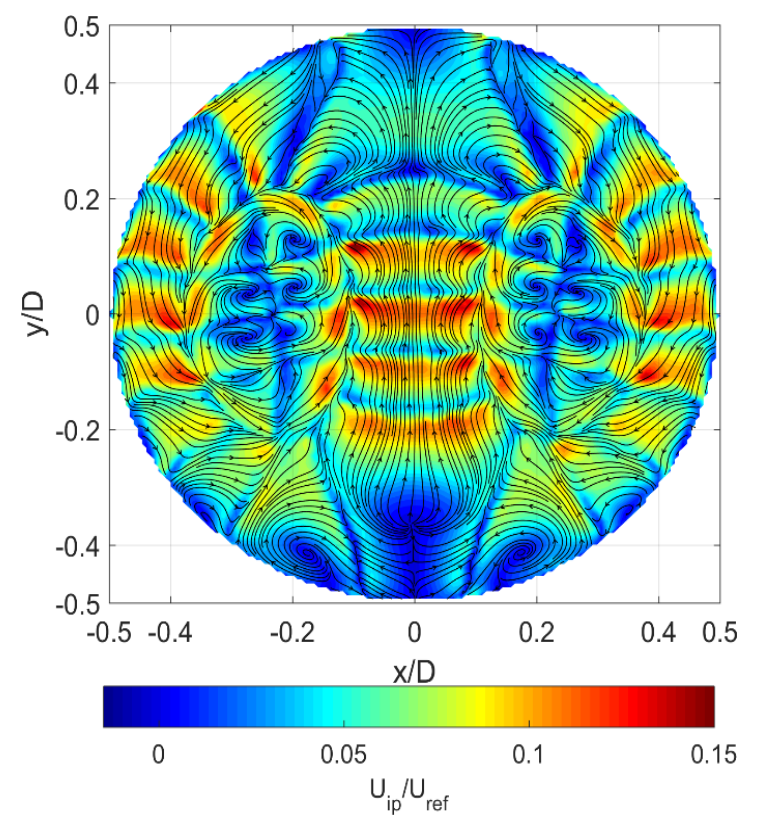

Figure 9. In-plane $(\mathrm{u}, \mathrm{v})$ normalized velocity close to outlet for Rev_C $(0.03 \mathrm{D}$ from TE)

As the flow progresses beyond $0.5 \mathrm{D}$, shown in Figure 10 for the Rev_C case, these smaller structures combine to a counter rotating vortex pair of a similar scale to the upstream structures. However, the magnitude of the secondary velocities of these structures are significantly reduced. 


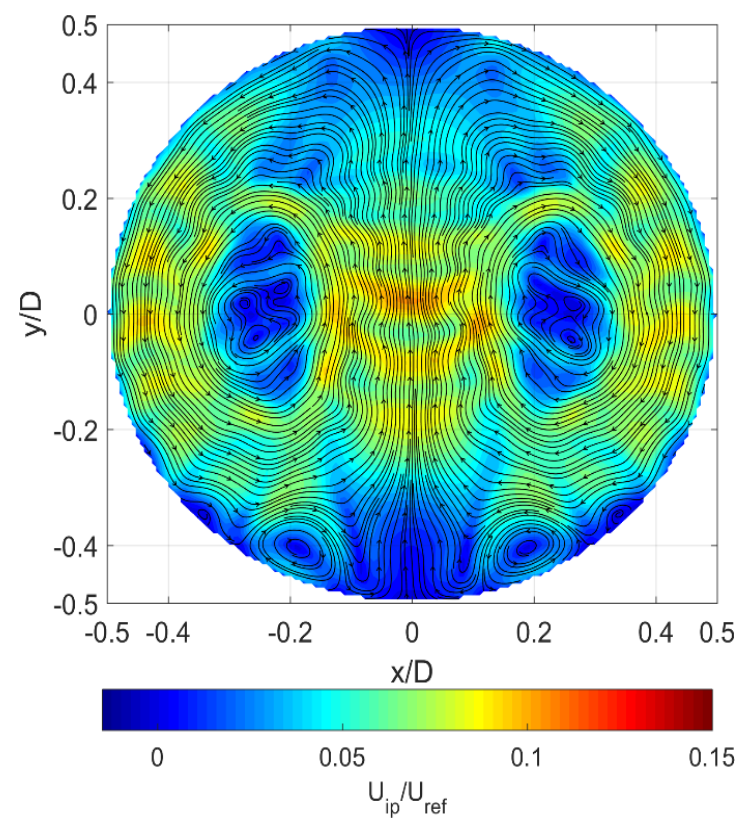

Figure 10. Developed in-plane (u,v) velocity for Rev_C $(\mathrm{z} / \mathrm{D}=0.5)$

Considering the turbulence development along the length of the duct (Figure 11), the presence of the vanepack has little effect overall. As earlier described, there is a region immediately downstream of the vanepack where the turbulence level is increased, however this is mixed out within $0.5 \mathrm{D}$.

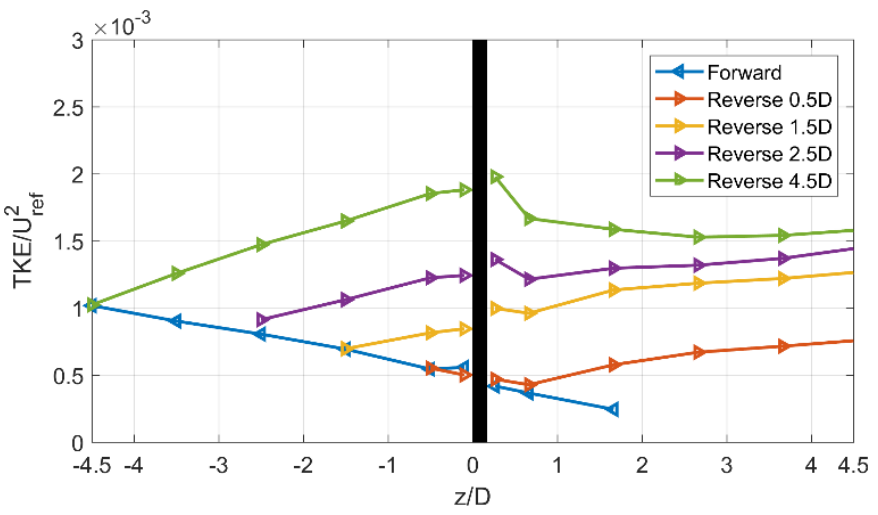

Figure 11. Development of area-weighted turbulent kinetic energy along duct (marker indicates flow direction)

In a similar approach to Figure 4, the total pressure drop coefficient, $\mathrm{K}$ is calculated for each of the reverse flow cases and is plotted together with the forward flow case for reference in Figure 12. In each case, the pressure drop coefficient is based on the relevant inlet plane for each flow case. Therefore, each starts at $\mathrm{K}=0$, and presents a similar ramp to the leading edge of the vanepack.

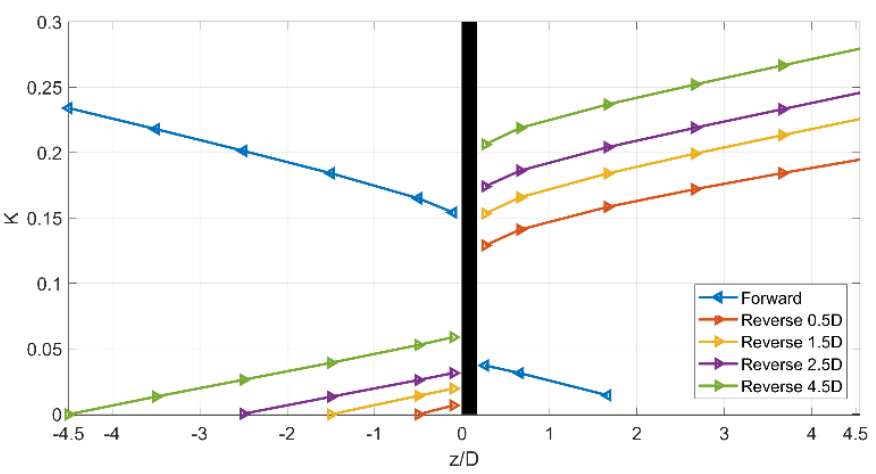

Figure 12. Pressure drop coefficient, K, based on mass-weighted averages (marker indicates flow direction)

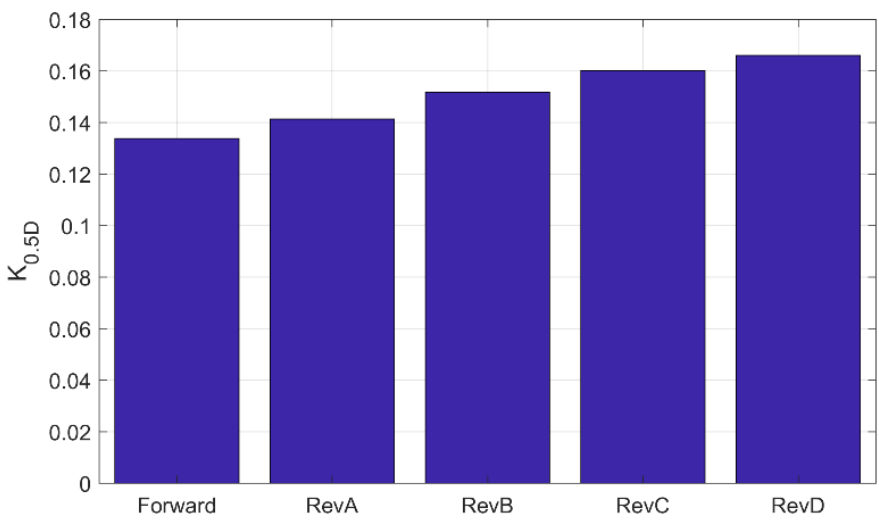

Figure 13. Comparison of vane-pack pressure drop coefficient, $\mathrm{K}$ evaluated at $0.5 \mathrm{D}$ up- and down- stream

For each reverse flow case, there is a pressure drop over the vanepack, after which the pressure continues to reduce slightly, again due to viscous losses. Figure 13 allows a visual comparison of the pressure drop for each flow condition.

Each of the reverse flow cases have a total pressure drop coefficient of less than $\mathrm{K}=0.18$, with the highest drop, exhibited in Rev_D which presents a $25 \%$ increase over the forward flow condition. The ranking order of the cases presented in Figure 13 shows that the further the inlet plane is from the vanepack, the greater the pressure drop. This can be attributed to the increased deviation of velocity distribution at the leading edge, as described in Figure 7.

Whilst the introduction of a vanepack for the purpose explored in this paper would come with a pressure drop penalty of up to $\mathrm{K}=0.18$, the resulting straighter flow entering a downstream component such as the SCR has the potential to result in a net saving. The evaluation of this net saving will form a part of the future work to be carried out.

\section{Conclusions}

This paper presents the concept of introducing an aerodynamic insert device, StreamVane, into a duct to assess the effectiveness of removing or reducing secondary velocities, i.e. swirl structures from the flow. This is relevant to flows within a TPS and, in particular, prior to components known to be sensitive to their aerodynamic boundary conditions. A device designed to introduce vorticity was mirrored and applied to a swirling flow with no further design modifications.

Page 7 of 9 
The initial analysis focuses on providing a baseline operation for the vanepack in the design condition, i.e. a uniform, axial inlet flow. The analysed showed that the design vorticity may be introduced as designed with a total pressure drop coefficient of $K=0.13$.

In reverse flow conditions a significant reduction in secondary flow velocity magnitude of $\sim 75 \%$ was observed. This demonstrates clear potential for a vanepack to be used to engineer a more uniform and straight flow for use around boundary condition sensitive components, thereby addressing the packaging problem described.

The sweep in inlet plane velocity distributions showed that the vanepack has robustness towards deviation around a nominal value. This is particularly of interest in a TPS where cyclic variation would be a signature of component inlet flow boundary. Consideration of the total pressure drop coefficient for each of these cases showed that as the velocity distribution differs further from that expected, the pressure drop coefficient increases. However, the device remains relatively low-pressure cost across the tested range.

As this particular device has been shown to be effective at reducing the magnitude of the counter-rotating vortex pair that it was designed for, it is feasible that the general StreamVane method may be extended to remove any particular secondary velocity profile of interest (such as the flow from a TPS system).

\section{Future Work}

The research presented in this paper represents the initial study of feasibility of the application of local aerodynamic inserts within automotive powertrains flows for the purpose of producing a uniform flow using a device that was not specifically designed for operation in this manner. Future work will:

1. Build on the current work to establish a robust design process that can be tailored to any given inlet flow. This could include an unsteady assessment.

2. Include the ability to remove (or tailor) bulk flow nonuniformity that may be generated in a TPS "packaging" such as by a bend.

3. Develop a vane pack for a specific application (SRC) and experimentally evaluate (i) the resultant flow field and (ii) the effect on said application - potentially in a low TRL rig. (iii) evaluate the net cost (pressure) saving across the subsystem, taking account of the $\mathrm{K}$ of the vanepack.

\section{References}

1. Senecal, P.K. and Leach, F., "Diversity in transportation: Why a mix of propulsion technologies is the way forward for the future fleet," Results Eng. 4(October):100060, 2019, doi:10.1016/j.rineng.2019.100060.

2. Automotive Council UK, "Thermal Propulsion Systems Roadmap," 2017.

3. Reitz, R.D., Ogawa, H., Payri, R., Fansler, T., Kokjohn, S., Moriyoshi, Y., Agarwal, A.K., Arcoumanis, D., Assanis, D., Bae, C., Boulouchos, K., Canakci, M., Curran, S., Denbratt, I., Gavaises, M., Guenthner, M., Hasse, C., Huang, Z., Ishiyama, T., Johansson, B., Johnson, T. V., Kalghatgi, G., Koike, M., Kong, S.C., Leipertz, A., Miles, P., Novella, R., Onorati, A., Richter, M., et al., "IJER

editorial: The future of the internal combustion engine," Int. J. Engine Res. 21(1):3-10, 2020, doi:10.1177/1468087419877990.

Zhao, D., Winward, E., Yang, Z., Stobart, R., and Steffen, T., "Characterisation, control, and energy management of electrified turbocharged diesel engines," Energy Convers. Manag. 135:416433, 2017, doi:10.1016/j.enconman.2016.12.033.

5. Yang, Z., Stobart, R., Lan, S., Mason, B., and Winward, E., "Towards Optimal Performance of a Thermoelectric Generator for Exhaust Waste Heat Recovery from an Automotive Engine," SAE Tech. Pap. 2018-April:1-8, 2018, doi:10.4271/2018-01-0050.

6. Qi, D.H., Chen, H., Geng, L.M., and Bian, Y.Z., "Experimental studies on the combustion characteristics and performance of a direct injection engine fueled with biodiesel/diesel blends," Energy Convers. Manag. 51(12):2985-2992, 2010, doi:10.1016/j.enconman.2010.06.042.

7. Merati, P., Mirhashemi, A., Fajardo-Hansford, C., and Liu, T. "Flow measurements in the exhaust system of a motorized engine," Int. J. Automot. Technol. 18(4):563-569, 2017, doi:10.1007/s12239017-0056-y.

8. Turner, C., Thornhill, D., McCullough, G., and Patel, S., "Comparison of Experimental PIV Data and CFD Simulations for Flow in a Diesel Particulate Filter Inlet Diffuser," SAE Int. J. Engines 4(1):1556-1570, 2011, doi:10.4271/2011-01-1241.

9. Persoons, T., Vanierschot, M., and Bulck, E. Van den, "Stereoscopic PIV measurements of swirling flow entering a catalyst substrate," Exp. Therm. Fluid Sci. 32(8):1590-1596, 2008 , doi:10.1016/j.expthermflusci.2008.04.011.

10. Persoons, T., Vanierschot, M., and Bulck, E. Van den, "Oblique inlet pressure loss for swirling flow entering a catalyst substrate," Exp. Therm. Fluid Sci. 32(6):1222-1231, 2008 , doi:10.1016/j.expthermflusci.2008.02.002.

11. Benjamin, S.F., Haimad, N., Roberts, C.A., and Wollin, J., "Modelling the flow distribution through automotive catalytic converters," Proc. Inst. Mech. Eng. Part C J. Mech. Eng. Sci. 215(4):379-383, 2001, doi:10.1243/0954406011520779.

12. Benjamin, S.F., Zhao, H., and Arias-Garcia, A., "Predicting the flow field inside a close-coupled catalyst - The effect of entrance losses," Proc. Inst. Mech. Eng. Part C J. Mech. Eng. Sci. 217(3):283-288, 2003, doi:10.1243/095440603762870036.

13. Guimaraes, T., Lowe, K.T., and O'Brien, W., “An overview of recent results using the StreamVane method for generating tailored swirl distortion in jet engine research," 54th AIAA Aerospace Sciences Meeting, 1-15, 2016, doi:10.2514/6.2016-0534.

14. Hoopes, K.M. and O'Brien, W.F., "The StreamVane Method: A New Way to Generate Swirl Distortion for Jet Engine Research," 111, 2013, doi:10.2514/6.2013-3665.

15. Lamb, H., "Hydrodynamics," 3rd ed., Cambridge University Press, Cambridge, 1906.

16. Chaplygin, S.A., "One case of vortex motion in fluid," Regul. Chaotic Dyn. 12(2):219-232, 2007 doi: $10.1134 / \mathrm{S} 1560354707020074$.

17. Gillespie, J., Lowe, K.T., and O’brien, W., "Definition of arbitrary swirl distortions by solutions to the helmholtz equation," AIAA Scitech 2019 Forum, ISBN 9781624105784, 2019, doi:10.2514/6.2019-1387.

18. Dunavant, J.C., "Cascade investigation of a related series of 6percent-thick guide-vane profiles and design charts," Natl. Advis Comm. Aeronaut., 1957.

Page 8 of 9 


\section{Contact Information}

Dr. Daniel Butcher

Department of Aeronautical \& Automotive Engineering, Loughborough University, Loughborough, UK

D.Butcher@lboro.ac.uk

John Gillespie

Kevin T. Crofton Department of Aerospace \& Ocean Engineering, Virginia Tech, Blacksburg, VA, USA

gillesj@vt.edu

Prof. Todd Lowe

Kevin T. Crofton Department of Aerospace \& Ocean Engineering, Virginia Tech, Blacksburg, VA, USA kelowe@vt.edu

Prof. Adrian Spencer

Department of Aeronautical \& Automotive Engineering, Loughborough University, Loughborough, UK

A.Spencer@lboro.ac.uk

\section{Acknowledgments}

LU authors gratefully acknowledge receipt of funding from the Advanced Propulsion Centre, APC for this work as part of the project 'System Optimisation Using Local Aerodynamic Modification (SOULAiM) - feasibility'. Assistance from Dr Apostolos Spanelis is gratefully acknowledged for his advice and assistance in the set-up of the numerical simulations 